McNally, R. J., Calamari, J. E., Hansen, P. M., et al (1988) Behavioural treatment of psychogenic polydipsia. Journal of Behavior Therapy and Experimental Psychiatry, 19, 57-61.

\& \& (1989) Preventing water intoxication: a reply. Journal of Behavior Therapy and Experimental Psychiatry, 20, 89-90.

John E. Calamari

Department of Psychology

University of Wisconsin-Whitewater

800 West Main Street

Whitewater

Wisconsin 53190-1790 USA

\section{Negative symptoms of schizophrenia}

SIR: I read with interest Phillips et al (Journal, August 1991, 159, 226-231) who reported that negative symptoms (as measured by SANS, Scale for the Assessment of Negative Symptoms) were prominent among both acute and chronic schizophrenics in China, and that during the acute phase they responded significantly to neuroleptic treatment. They therefore recommended affirmatively that negative symptoms "were an integral part of all stages of schizophrenia", and "should be understood more clearly by clinicians who must include their systematic evaluation in routine clinical examinations and consider their management in standard treatment protocols for schizophrenic patients". We do not, however, see how such recommendations may be clinically useful.

Negative symptoms were poorly defined clinical entities (Kulhara \& Chadda, 1987) until standard rating scales, which vary among themselves as to what they actually measure (de Leon et al, 1989), became available to make them measurable entities for researchers. Despite this, we find it clinically difficult to discern the significance of negative symptoms among acute schizophrenic in-patients. Some of the items on the SANS, e.g. poor eye contact, decreased movement, blocking, unsatisfactory grooming and decrease in sexual or recreational interest appear to be multifactorial constructs that can occur, in particular, as secondary reactions to a relapse of positive symptoms. Another SANS item, namely inattentiveness, has actually been correlated to positive symptoms (de Leon et al, 1989). Further, a negative correlation between negative and positive symptoms previously found by Andreason has not in fact been replicated by others (de Leon $e t$ al, 1989).

Aetiologically, if negative symptoms during acute schizophrenia are strongly related or even secondary to positive rather than the 'residual' negative symptoms that we commonly recognise, then their good response to neuroleptics becomes understandable. Indirect support for this is provided by the positive correlation sometimes found between negative and positive symptoms (de Leon et al, 1989), and by Kay et al (1986) who showed that the genealogical and predictive implications of negative symptoms were phase-specific, with those occurring during the acute phase indicating a good prognosis. Therefore, even if phenomenologically similar, negative symptoms during acute and chronic schizophrenia may be aetiologically, therapeutically and prognostically different entities. If this is so, it becomes arguable whether it is useful to lump them under the same term and conclude, as Phillips et al did, that negative symptoms respond to neuroleptics and therefore "the schizophrenic type is mutable". Instead, as the study had an impressively large sample of patients, it might be useful to correlate the two apparently different types of negative symptoms (i.e. phenomenotypes) with genetic or neurobiological factors (i.e. biotypes) in order to establish their validity in the complex schizophrenic syndrome.

It is important to stress that negative symptoms are non-specific, and may occur in neurosis, personality disorder and even healthy individuals. In particular, there is a great deal of resemblance between negative symptoms and the behavioural manifestations of depression, so that depressed patients can actually score higher than schizophrenics on the SANS (Kulhara \& Chadda, 1987). Even developers of negative symptoms scales have astutely recognised that for acute young schizophrenics, depression rather than negative symptoms is being measured (de Leon et al, 1989). Phillips et al, however, made no attempt at all to assess depression which might have confounded the evaluation of negative symptoms, despite the fact that electroconvulsive therapy was administered to $1 / 6$ of the patients.

Clincians have, over the decades, been confused by the ever-changing systems of classifying schizophrenic symptoms suggested by research experts. Today, they consider residual negative symptoms usually after the acute psychotic phase is over because of their empirical management and prognostic import. While negative symptoms, as measured during acute schizophrenia, may be of interest to researchers despite their questionable status, clinicians should perhaps not be burdened with this until better proof is available. In any case, Kraepelin in Dementia Praecox and Paraphrenia was probably not referring to them.

Kay, S. R., Fiszbein, A., Lindenmayer, J. P., et al (1986) Positive and negative syndromes in schizophrenia as a function of chronicity. Acta Psychiatrica Scandinavica, 74, 507-518. 
Kulhara, P. \& Chadda, R. (1987) Study of negative symptoms in schizophrenia and depression. Comprehensive Psychiatry, 28, 229-235.

de LeON, J., WILsON, W. H. \& Simpson, G. M. (1989) Measurement of negative symptoms in schizophrenia. Psychiatric Development 3, 211-234.

SING LeE

Prince of Wales Hospital

HELEN LEUNG

Shatin, $N T$

Hong Kong

\section{Hospital Anxiety and Depression Scale}

SIR: Chaturvedi (Journal, August 1991, 159, 298) claims that in his clinical study the Hospital Anxiety and Depression Scale (HAD Scale) could 'hardly discriminate' between anxiety and depression, and recommends that the two subscales be combined into a single score. Chaturvedi does not state whether he was using the HAD Scale in English in his Indian subjects, or a translated version; nor whether he evaluated the linguistic, conceptual or scale equivalence (Marsella, 1987) of the HAD Scale in the Indian setting.

With colleagues in Lahore (Pakistan), I have considerable experience with the HAD Scale both in English and in our Urdu version (Mumford et al, $1991 a$ ). The Urdu version is to be preferred unless the subject is very fluent in English: people generally express their emotional state better in their mothertongue. In one study, medical out-patients in Lahore completed the HAD Scale in Urdu, and DSM-III-R diagnoses were made independently at interview (Mumford et al, 1991b). Among 14 patients with depressive disorders, the mean HAD Scale anxiety score was 12.9 and HAD Scale depression score was 9.4; among 17 patients with anxiety and panic disorders the scores were 11.2 and 6.9; and among 23 patients with no psychiatric diagnosis they were 6.8 and 4.9 respectively.

These results are consistent with Chaturvedi's findings, but not with his conclusions. The HAD Scale depression (anhedonia) subscores were highest in the depressed patients, the most important clinical group to screen for; after excluding these patients, the HAD Scale anxiety subscores then successfully identified the anxiety and panic disorders. (High levels of anxiety among patients with depressive disorders is a common clinical finding.) In the whole sample, the HAD Scale anxiety items consistently correlated more highly with the anxiety subscore than they did with the depression subscore; and vice versa with the HAD Scale depression items.
Admittedly, the two subscales of the HAD Scale are not similarly calibrated and may require different cut-off scores for maximum screening efficiency. The fact that the authors have given two cut-off points (for possible and probable clinical significance) underlines the arbitrariness of any threshold when traits are continuously distributed in a population. However using the 10/11 cut-off on both the anxiety and the depression subscales, we found that the HAD Scale identified patients with DSM-III-R disorders in Lahore medical clinics with a sensitivity of $83 \%$ and specificity of $78 \%$.

The HAD Scale does not discriminate perfectly between anxiety and depression partly because it only taps the subjective component of the mood disorders. The HAD Scale was designed as a brief screening instrument for medical patients with concurrent physical illness. It is not a comprehensive inventory of anxiety or depressive symptoms and signs; it should not be used alone to make a clinical diagnosis, but only to indicate the probability of a psychiatric disorder being present. In my view, the HAD Scale fulfils this function satisfactorily both in Britain and in Pakistan.

Marsella, A. J. (1987) The measurement of depressive experience and disorder across cultures. In The Measurement of Depression (ed. A. J. Marsella). New York: Guilford Press.

MUMford, D. B., TAREen, I. A. K., BAJWA, M. A. Z., el al (1991a) The translation and evaluation of an Urdu version of the Hospital Anxiety and Depression Scale. Acta Psychiatrica Scandinavica, 83, 81-85.

— - - BнАтT, M. R., et al (1991b) An investigation of 'functional' somatic symptoms among patients attending hospital medical clinics in Pakistan: II. Using somatic symptoms to identify patients with psychiatric disorders. Journal of Psychosomatic Research, 35, 257-264.

Department of Psychiatry

DAvid B. Mumford

University of Leeds

25 Hyde Terrace

Leeds LS2 9LT

\section{Neuroleptic Malignant Syndrome}

SIR: We read with interest White \& Robins' reply to our letter (Journal, June 1991, 158, 858-859), Dalkin \& Kennedy's views on catatonia and neuroleptic malignant syndrome (NMS) (Journal, June 1991, $158,859)$ and Otani $e t$ al's "Is the predisposition to neuroleptic malignant syndrome genetically transmitted?" (Journal, June 1991, 158, 850-853).

Dr Otani's cases illustrate the diagnostic dilemma that Drs White \& Robins expressed about catatonia and NMS. In the three cases of a mother and her two daughters who were diagnosed to have NMS, the mother had an episode while not on neuroleptics and 